\title{
MATHEMATICAL MODELLING OF CANCER INVASION OF TISSUE: THE ROLE AND EFFECT OF NONLOCAL INTERACTIONS
}

\author{
ZUZANNA SZYMAŃSKA \\ ICM, University of Warsaw, \\ Pawińskiego $5 a$ \\ 02-106 Warszawa, Poland \\ mysz@icm.edu.pl
}

CRISTIAN MORALES RODRIGO

Institute of Applied Mathematics and Mechanics, Faculty of Mathematics, Informatics and Mechanics, University of Warsaw, ul. Banacha 2, 02-097 Warszawa, Poland

cristianmatematicas@yahoo.com

MIROSŁAW LACHOWICZ

Institute of Applied Mathematics and Mechanics, Faculty of Mathematics, Informatics and Mechanics, University of Warsaw, ul. Banacha 2, 02-097 Warszawa, Poland

lachowic@mimuw.edu.pl

MARK A.J. CHAPLAIN

The SIMBIOS Centre, Division of Mathematics, University of Dundee, 23 Perth Road, Dundee, DD1 $4 H N$, Scotland

chaplain@maths.dundee.ac.uk

In this paper we consider a mathematical model of cancer cell invasion of tissue (extracellular matrix). Two crucial components of tissue invasion are (i) cancer cell proliferation, and (ii) over-expression and secretion of proteolytic enzymes by the cancer cells. The proteolytic enzymes are responsible for the degradation of the tissue, enabling the proliferating cancer cells to actively invade and migrate into the degraded tissue. Our model focuses on the role of nonlocal kinetic terms modelling competition for space and degradation. The model consists of a system of reaction-diffusion-taxis partial differential equations, with nonlocal (integral) terms describing the interactions between cancer cells and the host tissue. We first of all prove results concerning the local existence, uniqueness and regularity of solutions of our system of nonlinear PDEs. We then extend these results to prove global existence, uniqueness and regularity of the solutions. Using Green's functions, we transform our original nonlocal equations into a coupled system of parabolic and elliptic equations and we undertake a numerical analysis of this equivalent system, presenting computational simulation results from our model showing travelling waves of cancer cells, degrading, invading and replacing the tissue. Finally, concluding 
remarks are made in the discussion section.

Keywords: cancer invasion of tissue; haptotaxis; nonlocal interactions; existence, uniqueness, regularity of solutions; computational simulations; travelling waves.

AMS Subject Classification: 35A05, 35A07, 35K55, 35K57, 35Q80, 65M99, 92C17

\section{Introduction}

The prognosis of a cancer is primarily dependent on its ability to invade and metastasize. Many steps that occur during tumour invasion and metastasis (as well as in a number of distinct physiological events in the healthy organism, such as trophoblast invasion, and skin wound healing) require a controlled and localized degradation of extracellular matrix (ECM) macromolecules. An even more localized degradation of matrix components is required when cells migrate through a basal lamina. The breakdown of these barriers is catalyzed by proteolytic enzymes released from the invading tumour. Most of these proteases belong to one of two general classes: many are metalloproteases, ${ }^{37}$ while others are serine proteases. ${ }^{7,8}$ Proteases give cancers their defining characteristic - the ability of malignant cells to break out of tissue compartments.

Cell migration plays a central role in a wide variety of physiological and pathophysiological processes, for instance embryo development, inflammation, and cancer metastasis (for reviews see Ref. 32). Cell migration is the locomotion of a cell on a substratum of extracellular matrix (ECM) proteins. Cells require attachment sites on extracellular matrices in order to reorganize their cytoskeleton and initiate protrusions important to migration. In this regard, cancer cells require a well-regulated, pericellular proteolysis to migrate. They must cleave linkages to the extracellular matrix and to other cells and degrade barriers like the basement membrane, the destruction of which is a common observation in invasive cancer as well as in normal pathological situations such as wound healing.

Although proteolysis and migration through tissue barriers are normal cell functions in specific physiological circumstances, it is clear that a general aspect of malignant neoplasms includes a shift toward sustained invasive capacity. For invasion to take place, cyclic attachment to matrix components and subsequent release must occur in a directed and controlled manner. This implies that proteolysis, although enhanced in tumour cells, is still tightly regulated in a temporal and spatial fashion with respect to cell attachment. Proteolytic activity is the balance between the local concentration of activated enzymes and their endogenous inhibitors.

Tumour cells encounter a variety of substratum-bound factors which may influence their directed migration at different stages in the process of tumour invasion and metastasis. Such factors can promote the directed movement of tumour cells by a mechanism termed haptotaxis. This is defined as cellular locomotion directed in response to a concentration gradient of a bound, non-diffusible molecule such 
as those present within the components of the extracellular matrix e.g. collagen, fibronectin, vitronectin. ${ }^{17,22}$ Such adhesive molecules can be present in spatially varying amounts within extracellular matrix. A cell that is constantly making and breaking adhesions with such molecules would move from a region of low concentration to an area where that adhesive molecule was more highly concentrated. Both cell-cell interactions and cell-matrix interactions play an important role during the invasive cascade. Connections through cell adhesion molecules, integrins, and cadherins stabilize tissue integrity, whereas loss or alteration of these cell surface proteins has been shown to be associated with increased metastatic potential. In this regard, pericellular proteolysis initiated by matrix degrading enzymes fulfils pivotal functions in cellular migration. Direct binding of molecules of matrix degrading enzymes to cell surface receptors or to extracellular matrix drastically increases the local concentration and the efficiency of protease formation/action.

In this paper we develop a new mathematical model of cancer cell invasion of tissue which focusses on the role of the highly controlled invasion mechanisms involved. In order to achieve this we develop an integro-differential equation model involving cancer cells and the tissue (extracellular matrix). The modelling of the highly localized degradation of tissue is achieved through the incorporation of a spatial kernel modeling the degradative interactions between cancer cells and the tissue. In the following section we formulate and describe the mathematical model which consists of two coupled, nonlinear parabolic partial integro-differential equations. In Section 3 using the theory of linear semigroups we first of all prove results concerning the local existence, uniqueness and regularity of solutions of our system of nonlinear PDEs. In Section 4, we then extend these results to prove global existence, uniqueness and regularity of the solutions. Using Green's functions, in Section 5 we transform our original nonlocal equations into a coupled system of parabolic and elliptic equations and we undertake a numerical analysis of this equivalent system, presenting computational simulation results from our model showing travelling waves of cancer cells, degrading, invading and replacing the tissue. Finally, concluding remarks are made in the Discussion Section 6.

\section{The Mathematical Model}

Previous mathematical models for cancer invasion and metastasis can be found in, for example, Refs. 5, 6, 16, 18, 19, 25, 31, 35, 38, 39. Many of these papers examine how cancer cells respond to ECM gradients via haptotaxis. The gradients are created through the degradation of the extracellular matrix (ECM) by matrix degrading enzymes (MDEs). In this paper, we will base our mathematical model on generic solid tumour growth, which for simplicity we assume is at an avascular stage, focussing initially solely on the interactions between the cancer cells and the surrounding tissue. We develop a mathematical model consisting of two coupled partial differential equations (PDEs) describing the evolution in time and space 
of the system variables and including nonlocal (integral) terms. The key physical variables are taken to be the cancer cell density (denoted by $u$ ) and the extracellular matrix protein density (denoted by $v$ ). The focus of the model is on examining different key features of the system separately i.e. cell random motility, haptotaxis, and proliferation and a specific emphasis on the highly controlled process of matrix degradation. In vivo matrix degradation is achieved either through re-binding of secreted MDE to receptors on the cancer cell surface, or through the secreted MDE diffusing a short distance in to the tissue where it is then bound and can activate other matrix degrading components such as plasmin. In either case, the actual region of degradation is highly localised around the leading edge invasive cancer cells. We model this effect by including an integral term in our PDEs. This approach means that we do not explicitly include an equation for the MDE.

We now describe the way in which the cancer cell density $u(t, x)$ and the extracellular matrix density $v(t, x)$ are involved in invasion and derive partial differential equations governing the evolution of each variable.

\section{(a) Cancer Cells:}

It is well known that pericellular proteolysis plays a crucial role in cancer cell invasion. The controlled degradation of the extracellular matrix by cancer cell associated proteases allows cancer cells to invade surrounding tissues and gain access to the circulation. ${ }^{2}$ In addition, invasive cells in vivo adhere to surrounding ECM molecules via specific receptors such as integrins or urokinase plasminogen activator receptors, and produce and secrete several types of matrix degrading enzyme (MDE) such as matrix metalloproteinases and urokinase plasminogen activators ${ }^{11}$. The actual matrix degradation is achieved largely through re-binding of MDE to receptors on the cancer cell surface producing a highly localized area of degradation. The consequent digestion of ECM allows the cells to move into the spaces thereby created and also sets up tissue gradients, which the cells then exploit to move forwards. ${ }^{10,12,33,34,43}$ Movement up concentration gradients of ECM has been reported as a mechanism enabling movement through tissues by a variety of cell types. Tumour cell motility toward high concentrations/densities of substratumbound insolubilized components has been termed "haptotaxis". The haptotaxis function in our model is denoted $\chi(v)$. Using the derivation from kinetic analysis of a model mechanism for the cell-surface-receptor-extracellular-ligand binding dynamics, we assume that this haptotactic function is of the form:

$$
\chi(v)=\frac{\chi_{u}}{\left(\alpha_{0}+\beta_{0} v\right)^{2}},
$$

where $\chi_{u} \geq 0$ and $\alpha_{0}, \beta_{0}>0^{41}$. Other possibilities for this function are given in Ref. 21. We assume that the cancer cells also migrate through random motility, with a (constant) coefficient $D_{u}$.

Individual cells proliferating within the overall tumour cell mass have to compete 
for nutrients, oxygen and space. So even cancer cells (that in culture may proliferate indefinitely) under some conditions are suppressed in their proliferation e.g. cells in the interior of a solid tumour do not divide as quickly as the cells on the surface (mainly due to the lack of space and nutrients). Describing cell growth we therefore have to take into account this phenomenon. It is possible to do this by using a logistic growth term, for instance. However, assuming ordinary logistic growth may well be a crude over-simplification, since it means that proliferation of the cells depends on the cells and the ECM concentration/density at given point, whereas the proliferation probably actually depends on the cell and ECM concentration/density in a local neighbourhood. The immediate surrounding of a cell influences its ability to divide and therefore we include a nonlocal term describing a neighbourhood of a cell that inhibits its proliferation in the model and we adopt the following proliferation term in our model:

$$
\mu_{1} u(t, x)\left(1-\int_{\Omega} k_{1,1}(x, y) u(t, y) \mathrm{d} y-\int_{\Omega} k_{1,2}(x, y) v(t, y) \mathrm{d} y\right),
$$

where $\Omega$ is a bounded domain in $\mathbb{R}^{d}(d \geq 1)$ with smooth boundary $\partial \Omega$, $\mu_{1}$ represents the cancer cell proliferation rate, and $k_{1,1}, k_{1,2}$ are given spatial kernels. The terms

$$
u(t, x) \int_{\Omega} k_{1,1}(x, y) u(t, y) \mathrm{d} y
$$

and

$$
u(t, x) \int_{\Omega} k_{1,2}(x, y) v(t, y) \mathrm{d} y,
$$

describe the inhibition of the cells' proliferation caused by the density of surrounding cells and ECM respectively.

Therefore the equation describing the dynamics of tumour cells reads

$$
\begin{aligned}
& \partial_{t} u(t, x)=\nabla \cdot\left(D_{u} \nabla u(t, x)\right)-\nabla \cdot(\chi(v) u(t, x) \nabla v(t, x)) \\
& +\mu_{1} u(t, x)\left(1-\int_{\Omega} k_{1,1}(x, y) u(t, y) \mathrm{d} y-\int_{\Omega} k_{1,2}(x, y) v(t, y) \mathrm{d} y\right)
\end{aligned}
$$

\section{(b) Extracellular Matrix}

We now turn attention to the extracellular matrix (ECM). This is known to contain many macromolecules such as vitronectin, laminin and fibronectin which can be degraded by several matrix degrading enzymes.

Since extracellular matrix (ECM) is "static", we neglect any random motion and focus solely on its degradation by the cancer cells. As mentioned above, matrix 
degradation in vivo is achieved either through re-binding of MDE to receptors on the cancer cell surface or by MDE-activation of other degrading components in the matrix. This has the effect of producing a region of degradation that is restricted to a small distance around the leading edge of the invading cancer cells. Therefore, in our model we assume that cancer cells themsleves degrade the ECM upon contact in a highly controlled and restricted manner, and use an integral term to capture this, thus simplifying our model slightly by not explicitly modelling the MDE. We also suggest that ECM components re-establish or re-model while they are competing for space with the invasive cells in a manner similar to that describing cancer cell proliferation. Thus, in the absence of cancer cells, extracellular matrix remodels in a logistic manner. On the other hand, the presence of cancer cells leads to competition for space between the cancer cells and the ECM which again we model by incorporating a crowding term into the logistic growth. Using a modified logistic growth with rate constant $\mu_{2}$ to describe the ECM production, and taking $\gamma$ to represent the rate of degradation, we have the following equation for the extracellular matrix:

$$
\begin{aligned}
& \partial_{t} v(t, x)=-\gamma v(t, x) \int_{\Omega} k(x, y) u(t, y) \mathrm{d} y \\
& +\mu_{2} v(t, x)\left(1-\int_{\Omega} k_{2,1}(x, y) u(t, y) \mathrm{d} y-\int_{\Omega} k_{2,2}(x, y) v(t, y) \mathrm{d} y\right),
\end{aligned}
$$

where $\gamma$ is a given nonnegative parameter and $k_{2,1}, k_{2,2}$ and $k$ are non-negatively defined functions.

The complete system of equations describing the interactions between the tumour cells and extracellular matrix is:

$$
\begin{aligned}
\partial_{t} u(t, x)= & \nabla \cdot\left(D_{u} \nabla u(t, x)\right)-\nabla \cdot(\chi(v) u(t, x) \nabla v(t, x)) \\
& +u(t, x) \mu_{1}\left(1-\int_{\Omega} k_{1,1}(x, y) u(t, y) \mathrm{d} y\right. \\
& \left.-\int_{\Omega} k_{1,2}(x, y) v(t, y) \mathrm{d} y\right) \\
\partial_{t} v(t, x)= & -\gamma v(t, x) \int_{\Omega} k(x, y) u(t, y) \mathrm{d} y \\
& +v(t, x) \mu_{2}\left(1-\int_{\Omega} k_{2,1}(x, y) u(t, y) \mathrm{d} y\right. \\
& \left.-\int_{\Omega} k_{2,2}(x, y) v(t, y) \mathrm{d} y\right),
\end{aligned}
$$

where $D_{u}, \mu_{1}, \mu_{2}, \gamma$ are given nonnegative parameters, $k, k_{i, j}(i, j=1,2)$ are given spatial kernels and $\chi$ is a function that depend on $v$. We assume that

$$
\begin{gathered}
k, k_{i, j} \in L^{\infty}, \quad \nabla k, \nabla k_{2, j} \in L^{\infty}, \quad i, j=1,2, \\
k \geq 0 \quad k_{i, j} \geq 0 \quad i, j=1,2 \\
\quad \chi \in C^{2}(\mathbb{R}), \quad \chi \geq 0 \\
\text { and } \chi, \chi^{\prime} \text { are globally Lipschitz continuous. }
\end{gathered}
$$


The system (2.4) may be rewritten in the following compact version

$$
\begin{aligned}
& \partial_{t} u=D_{u} \Delta u-\nabla \cdot(u \chi(v) \nabla v)+\mu_{1} u\left(1-k_{1,1} \circledast u-k_{1,2} \circledast v\right), \\
& \partial_{t} v=-\gamma v k \circledast u+\mu_{2} v\left(1-k_{2,1} \circledast u-k_{2,2} \circledast v\right),
\end{aligned}
$$

where $k \circledast u(x)=\int_{\Omega} k(x, y) u(y) \mathrm{d} y$.

Remark 2.1. If instead of $\Omega$ we consider $\mathbb{R}^{d}$ or $d$-dimensional torus $\mathbb{T}^{d}$ (periodic boundary conditions), then it is natural to use the convolution $\star$ instead of $\circledast$.

Boundary Conditions: Guided by the in vitro experimental protocol in which invasion takes place within an isolated system, we assume that there is no-flux of cancer cells on the boundary of the domain, i.e.

$$
\left.u \chi(v) \frac{\partial v}{\partial \nu}-D_{u} \frac{\partial u}{\partial \nu}=0 \quad \text { on } \quad\right] 0, T[\times \partial \Omega
$$

where $\nu$ is the outward normal vector to $\partial \Omega$.

Initial Conditions: We consider the initial-boundary-value problem: Eqs. (2.8), (2.9) together with the initial data

$$
(u(0, x), v(0, x))=\left(u_{0}(x), v_{0}(x)\right) .
$$

\section{Local existence}

Denote the norm of $L^{p}(\Omega)$ by $\|\cdot\|_{p}$ and the norm of the Sobolev space $W^{l, p}(\Omega)$ by $\|\cdot\|_{p}^{(l)}$. Let

$$
p>d
$$

For a fixed $T>0$ let

$$
\|u\|_{p}=\sup _{t \in[0, T]}\|u(t)\|_{p}, \quad\|u\|_{p}^{(l)}=\sup _{t \in[0, T]}\|u(t)\|_{p}^{(l)}
$$

The positive dimensionless parameters $D_{u}, \gamma, \mu_{1}, \mu_{2}$ do not play essential rôle in the analysis and therefore from now on we assume that

$$
D_{u}=\gamma=\mu_{1}=\mu_{2}=1
$$

Now we introduce the new variable (see Ref. 21 and references therein)

$$
w(t, x)=\frac{u(t, x)}{z(t, x)}, \quad z(t, x)=\exp \left(\int_{0}^{v(t, x)} \chi(s) \mathrm{d} s\right)
$$


In the new variables the equation reads

$$
\begin{aligned}
\partial_{t} w= & \Delta w+\chi(v) \nabla v \cdot \nabla w+w\left(1-k_{1,1} \circledast(w z)-k_{1,2} \circledast v\right)+ \\
& +\chi(v) w v k \circledast(w z)-\chi(v) w v\left(1-k_{2,1} \circledast(w z)-k_{2,2} \circledast v\right) \\
\partial_{t} v= & -v k \circledast(w z)+v\left(1-k_{2,1} \circledast(w z)-k_{2,2} \circledast v\right)
\end{aligned}
$$

on $] 0, T[\times \Omega$, with the boundary conditions

$$
\left.\frac{\partial w}{\partial \nu}=0, \quad(t, x) \in\right] 0, T[\times \Omega,
$$

and the intial data

$$
(w, v)(0, x)=\left(w_{0}, v_{0}\right)(x), \quad x \in \Omega .
$$

In order to show local existence of solutions to (3.4)-(3.6), we apply the theory of linear semigroups. Let $A_{p}$ denote the sectorial operator defined by

$$
A_{p} u=-\Delta u, \quad u \in D\left(A_{p}\right)=\left\{\xi \in W^{2, p}(\Omega): \quad \frac{\partial \xi}{\partial \nu}=0 \text { on } \partial \Omega\right\} .
$$

Since $\Re D\left(A_{p}+1\right)=1>0$, where $D\left(A_{p}+1\right)$ is the spectrum of $A_{p}+1$, the operator $A_{p}+1$ possesses the fractional powers $\left(A_{p}+1\right)^{\beta}, \beta \geq 0$. Let $X_{p}^{\beta}=$ $D\left(\left(A_{p}+1\right)^{\beta}\right)$ then we have the following embedding properties ${ }^{27}$ (Theorem 1.6.1)

$$
\begin{array}{ll}
X_{p}^{\beta} \hookrightarrow W^{k, q}(\Omega) & \text { for } k-\frac{d}{q}<2 \beta-\frac{d}{p}, \quad q \geq p>d \\
X_{p}^{\beta} \hookrightarrow C^{\kappa}(\bar{\Omega}) & \text { for } 0 \leq \kappa<2 \beta-\frac{d}{p},
\end{array}
$$

where $C^{\kappa}$ is the space of $[\kappa]$-times continuously differentiable functions with the $[\kappa]$-order derivative satisfying the Hölder condition with exponent $\kappa-[\kappa]$.

Since $A_{p}+1$ is a sectorial operator then $\left\{e^{-t\left(A_{p}+1\right)}\right\}_{t \geq 0}$ defines an analytical semigroup. Moreover for $u \in L^{p}(\Omega)$ we have (see Ref. 27)

$$
\left\|\left(A_{p}+1\right)^{\beta} e^{-t\left(A_{p}+1\right)} u\right\|_{p} \leq c t^{-\beta} e^{-\delta t}\|u\|_{p}
$$

where $\delta \in] 0,1[$.

Let $p>d$ and $\beta \in] \frac{1}{2}+\frac{d}{2 p}, 1\left[\right.$ be fixed. We denote the spaces $X_{p}^{\beta}$ by $X$ and the corresponding norm by $\|\cdot\|$. Given $T>0$, let

$$
Y=C^{0}([0, T] ; X), \quad Y^{1, \infty}=C^{0}\left([0, T] ; W^{1, \infty}(\Omega)\right)
$$

with the norms denoted by $\|\cdot \mid\|$ and $\|\cdot\| \|_{\infty}^{(1)}$, respectively.

Now the local existence theorem can be formulated

Theorem 3.1. Let initial data (3.6) be such that $\left(w_{0}, v_{0}\right) \in X \times W^{1, \infty}(\Omega)$. If assumptions (2.5), (2.7) are satisfied, then there exists $T>0$ such that problem (3.4)-(3.6) has a unique solution $(w, v)$ in $Y \times Y^{1, \infty}$ and

$$
\begin{aligned}
& w \in C^{1}(] 0, T[; X) \cap C^{0}(] 0, T\left[; W^{2, p}(\Omega)\right), \\
& v \in C^{1}(] 0, T\left[; W^{1, \infty}(\Omega)\right) .
\end{aligned}
$$


Moreover, if $w_{0}, v_{0} \geq 0$, then

$$
w(t) \geq 0, \quad v(t) \geq 0, \quad t \in[0, T] .
$$

Let $T_{\max }$ be the maximal existence time. If there is a continuous function $\omega$ : ] $0, \infty[\rightarrow] 0, \infty[$ such that, for each $\tau>0$,

$$
\|w(t)\| \leq \omega(\tau), \quad\|v(t)\|_{\infty}^{(1)} \leq \omega(\tau) \quad 0<t<\min \left\{\tau, T_{\max }\right\}
$$

then $T_{\max }=+\infty$.

Proof. Let $B_{R}$, for some $R>0$, be the ball

$$
B_{R}=\left\{(w, v) \in Y \times Y^{1, \infty}: \quad\left\|w-w_{0}\right\|+\left\|v-v_{0}\right\|_{\infty}^{(1)} \leq R\right\}
$$

and $J=\left(J_{1}, J_{2}\right)$ be the operator

$$
\begin{aligned}
J_{1}(w, v)= & e^{-t\left(A_{p}+1\right)} w_{0}+\int_{0}^{t} e^{-(t-s)\left(A_{p}+1\right)}(\chi(v) \nabla v \cdot \nabla w+2 w \\
& -w k_{1,1} \circledast(w z)-w k_{1,2} \circledast v+\chi(v) w v k \circledast(w z) \\
& \left.-\chi(v) w v\left(1-k_{2,1} \circledast(w z)-k_{2,2} \circledast v\right)\right) \mathrm{d} s \\
J_{2}(w, v)= & v_{0}+\int_{0}^{t}(-v k \circledast(w z) \\
& \left.+v\left(1-k_{2,1} \circledast(w z)-k_{2,2} \circledast v\right)\right) \mathrm{d} s .
\end{aligned}
$$

Fix $R>0$. We first prove that $B_{R}$ is invariant under $J$ if $T>0$ is sufficiently small. Since $w_{0} \in X$ and using inequality (3.8) we obtain

$$
\begin{aligned}
\left\|J_{1}-w_{0}\right\| & \leq \frac{R}{2}+\operatorname{const} \int_{0}^{t}(t-s)^{-\beta} e^{-\delta(t-s)}\left(\|\chi(v)\|_{\infty}\|\nabla w\|_{\infty}\|\nabla v\|_{\infty}\right. \\
& +2\|w\|_{\infty}+\|w\|_{\infty}\left\|k_{1,1} \circledast(w z)\right\|_{p}+\|w\|_{\infty}\left\|k_{1,2} \circledast v\right\|_{p} \\
& +\|\chi(v)\|_{\infty}\|w\|_{\infty}\|v\|_{\infty}\left(\|k \circledast(w z)\|_{p}+1\right. \\
& \left.\left.+\left\|k_{2,1} \circledast(w z)\right\|_{p}+\left\|k_{2,2} \circledast v\right\|_{p}\right)\right) \mathrm{d} s .
\end{aligned}
$$

By the definition of $p$ and $\beta$ we may choose $\kappa=1$ in $(3.7)_{2}$ and therefore by $(2.5)$ we obtain

$$
\begin{aligned}
\left\|J_{1}-w_{0}\right\| & \leq \frac{R}{2}+\frac{\text { const }}{1-\beta} T^{1-\beta}\|w\|\left(\left(\|v\|_{\infty}+\chi_{0}\right)\|v\| \|_{\infty}^{(1)}+1\right. \\
& +\|w\|\left\|_{p}\right\| z\left\|_{\infty}+\right\| v\|\|_{p}+\|v\|_{\infty}\|w\|\left\|_{p}\right\| z \|_{\infty} \\
& \left.\left.+\left(\|v\|_{\infty}+\chi_{0}\right)\right)\|v\|_{\infty}\left(1+\|w\|_{p}\|z\|_{\infty}+\|v\|_{p}\right)\right),
\end{aligned}
$$

where $\chi_{0}$ is a positive constant. 
Assuming now that $(w, v) \in B_{R}$, by (3.7) and (3.14) we have

$$
\left\|J_{1}(w, v)-w_{0}\right\| \leq \frac{R}{2}+\frac{\text { const }}{1-\beta} T^{1-\beta},
$$

where the constant indicated by "const" depends on $R,\left\|w_{0}\right\|$, and $\|v\|_{\infty}^{(1)}$.

In the same manner, by (2.5) and (2.7), we obtain

$$
\left\|J_{2}(w, v)-v_{0}\right\|_{\infty}^{(1)} \leq \frac{R}{2}+\operatorname{const} T,
$$

where the constant indicated by "const" depends on $R,\left\|w_{0}\right\|$, and $\|v\|_{\infty}^{(1)}$. Hence we can choose $T$ sufficiently small to assert that $J\left(B_{R}\right) \subset B_{R}$.

Similar arguments show that

$$
\left\|J_{1}\left(w_{1}, v_{1}\right)-J_{1}\left(w_{2}, v_{2}\right)\right\| \leq \frac{\text { const }}{1-\beta} T^{1-\beta}\left(\left\|w_{1}-w_{2}\right\|\|+\| v_{1}-v_{2} \|_{\infty}^{(1)}\right)
$$

and

$$
\left\|J_{2}\left(w_{1}, v_{1}\right)-J_{2}\left(w_{2}, v_{2}\right)\right\|_{\infty}^{(1)} \leq \operatorname{const} T\left(\left\|w_{1}-w_{2}\right\|+\left\|v_{1}-v_{2}\right\|_{\infty}^{(1)}\right),
$$

where the constants indicated by "const" depend on $R,\left\|w_{0}\right\|$, and $\|v\|_{\infty}^{(1)}$.

Hence given $T$ small enough we obtain the contractivity of the operator $J$ in $B_{R}$. Thus local existence and uniqueness follow.

Now, we proceed with the proof of (3.9). Let $\left.t_{0} \in\right] 0, T$ [ fixed, then (see Ref. 27, Lemma 3.5.2) entails

$$
\frac{d}{d t} u\left(t_{0}, \cdot\right) \in X_{p}^{\gamma}
$$

for any $\gamma<1$. Next, we rewrite the first equation of (3.4) in the following form

$$
-\Delta w-b \cdot \nabla w+w=f-\frac{\partial w}{\partial t}
$$

where $b=\chi(v) \nabla v \in\left(L^{\infty}(\Omega)\right)^{N}$ and

$f=w\left(2-k_{1,1} \circledast(w z)-k_{1,2} \circledast v\right)+\chi(v) w v\left(k \circledast(w z)-1+k_{2,1} \circledast(w z)+k_{2,2} \circledast v\right) \in L^{p}(\Omega)$.

Therefore, from the Schauder estimates we get $q\left(t_{0}\right) \in W^{2, p}(\Omega)$, ending the proof of (3.9).

From (2.5), (2.7) and the regularity of our solutions we obtain

$$
\left(1-k_{1,1} \circledast(w z)-k_{1,2} \circledast v+\chi(v) v\left(k \circledast(w z)-1+k_{2,1} \circledast(w z)+k_{2,2} \circledast v\right)\right) \in L^{\infty}(] 0, T[\times \Omega) .
$$

Consequently the non-negativity of $w$ follows from the maximum principle arguments, see Ref. 3 (page 18), or Ref. 4 (Section 15). Next we observe that the equation for $v$ can be written as

$$
v_{t}=v f,
$$


with $f=1-k \circledast(w z)-k_{2,2} \circledast v-k_{2,1} \circledast(w z)$. Thus,

$$
v(x, t)=v_{0}(x) e^{\int_{0}^{t} f(x, s) d s},
$$

concluding the non-negativity of $v$.

The last statement follows by prolongation arguments (see Ref. 27, Theorem 3.3.4). This finishes the proof.

Remark 3.1. Theorem 3.1 yields existence, uniqueness and regularity of solutions to problem (2.8), (2.9), (2.10).

Remark 3.2. By using the Fourier transform techniques one may prove an analogous local existence and uniqueness theorem for Eq. (2.4) with (3.6), $\chi \equiv$ const, in the whole space $\mathbb{R}^{d}$, with the convolution $\star$ instead of $\circledast$, with

$$
u(t) \in H^{l}\left(\mathbb{R}^{d}\right), \quad v(t) \in H^{s}\left(\mathbb{R}^{d}\right), \quad t \in[0, T],
$$

where $T>0$ is sufficiently small, $l>2+\frac{d}{2}$, and $s>l+1+\frac{d}{2}$.

\section{Global existence}

It is very well-known that for the (local) Keller-Segel-type chemotaxis equations (see Refs. 21, 28, 29, 30) the solutions may blow up in finite time. Here we prove that the solutions to the nonlocal equation (2.4) exist globally in any space dimension $d$ without imposing any kind of smallness conditions in the initial conditions.

In this section we assume that $(u, v)$ is a nonnegative solution to Eq. (2.4), (2.9), (2.10) given by Theorem 3.1 - see Remark 3.1 - on the time interval $[0, T]$, with $T>0$. We start with some simple lemmas that deliver a priori estimates.

\section{Lemma 4.1.}

$$
v(t, x) \leq v_{0}(x) e^{T}, \quad t \in[0, T], \quad x \in \Omega .
$$

Proof. The statement is a consequence of the nonnegativity of $u$ and $v$, the assumption (2.6) as well as the inequality

$$
\partial_{t} v \leq v
$$

that follows from Eq. (2.4).

\section{Lemma 4.2 .}

$$
\|u\|_{1} \leq\left\|u_{0}\right\|_{1} e^{T}, \quad\|\| w\left\|_{1} \leq\right\| u_{0} \|_{1} e^{T} .
$$

Proof. Integrating Eq. $(2.4)_{1}$ we obtain

$$
\begin{aligned}
& \|u(t, .)\|_{1}=\int_{\Omega} u(t, x) \mathrm{d} x \\
& \leq \int_{\Omega} u_{0}(x) \mathrm{d} x+\int_{0}^{t} \int_{\Omega} u\left(t^{\prime}, x\right) \mathrm{d} x \mathrm{~d} t^{\prime} \\
& \left\|u_{0}\right\|_{1}+\int_{0}^{t}\left\|u\left(t^{\prime}, .\right)\right\|_{1} \mathrm{~d} t^{\prime} .
\end{aligned}
$$


Hence, by Gronwall's lemma

$$
\|u\|_{1} \leq\left\|u_{0}\right\|_{1} e^{T},
$$

Taking into account that $w=\frac{u}{z}$ and $z^{-1} \leq 1$ yields (4.1).

\section{Lemma 4.3.}

$$
\|v\|_{\infty}^{(1)} \leq \mathfrak{c}_{1}\left(\left\|v_{0}\right\|_{\infty}^{(1)}+\mathfrak{c}_{2}\right)
$$

where the constants $\mathfrak{c}_{1}$ and $\mathfrak{c}_{2}$ depend on $T,\left\|u_{0}\right\|_{1}$ and $\left\|v_{0}\right\|_{\infty}$.

Proof. By Eq. (3.4) $)_{2}$ we have

$$
\begin{aligned}
\partial_{t} \nabla v= & -\nabla v k \circledast u-v\left(\nabla_{1} k\right) \circledast u \\
& +\nabla v\left(1-k_{2,1} \circledast u-k_{2,2} \circledast v\right) \\
& +v\left(\left(\nabla_{1} k_{2,1}\right) \circledast u-\left(\nabla_{1} k_{2,2}\right) \circledast v\right),
\end{aligned}
$$

where by $\nabla_{1}$ we indicate the gradient with respect to the first variable $x$. Therefore, by (2.5), we obtain

$$
\begin{aligned}
|\nabla v(t, x)| \leq & \left|\nabla v_{0}(x)\right|+\operatorname{const} \int_{0}^{t}\|v(s)\|_{\infty}\left(\|u(s)\|_{1}+\|v(s)\|_{\infty}\right) \\
& +\operatorname{const} \int_{0}^{t}|\nabla v(s, x)|\left(1+\|u(s)\|_{1}+\|v(s)\|_{\infty}\right) .
\end{aligned}
$$

By (4.1) and (4.3) it follows

$$
\begin{aligned}
|\nabla v(t, x)| \leq & \left(\left|\nabla v_{0}(x)\right|+\operatorname{const} T e^{T}\left\|v_{0}\right\|_{\infty}\left(\left\|u_{0}\right\|_{p}+\left\|v_{0}\right\|_{\infty}\right)\right) \\
& \times \exp \left(\operatorname{const} T e^{T}\left(1+\left\|u_{0}\right\|_{p}+\left\|v_{0}\right\|_{\infty}\right) .\right.
\end{aligned}
$$

This completes the proof.

\section{Lemma 4.4.}

$$
\|w\| \leq \mathfrak{c}_{3}\left\|w_{0}\right\|
$$

where the constants $\mathfrak{c}_{3}$ depends on $T,\left\|u_{0}\right\|_{1}$ and $\left\|v_{0}\right\|_{\infty}$.

Proof. Estimates similar to that in (3.13) show

$$
\begin{aligned}
\|w(t)\| & \leq \text { const } t^{-\beta} e^{-\delta t}\left\|w_{0}\right\|_{p}+\text { const } \int_{0}^{t}(t-s)^{-\beta} e^{-\delta(t-s)}\|w\| \\
& \times\left(1+\|\chi(v)\|_{\infty}\|\nabla v\|_{\infty}+\left\|k_{1,1} \circledast u\right\|_{p}+\left\|k_{1,2} \circledast v\right\|_{p}\right. \\
& +\|v\|_{\infty}\|\chi(v)\|_{\infty}\left(\|k \circledast u\|_{p}+1\right. \\
& \left.\left.+\left\|k_{2,1} \circledast u\right\|_{p}+\left\|k_{2,2} \circledast v\right\|_{p}\right)\right) \mathrm{d} s .
\end{aligned}
$$


Thus, by (2.5)

$$
\begin{aligned}
\|w(t)\| & \leq \text { const } t^{-\beta}+\operatorname{const} \int_{0}^{t}(t-s)^{-\beta}\|w(s)\| \\
& \times\left(1+\left(\|v(s)\|_{\infty}^{(1)}+\chi_{0}\right)\|v(s)\|_{\infty}^{(1)}+\|u(s)\|_{1}+\|v(s)\|_{\infty}\right. \\
& \left.+\left(\|v(s)\|_{\infty}+\chi_{0}\right)\|v(s)\|_{\infty}\left(\|u(s)\|_{1}+1+\|v(s)\|_{\infty}\right)\right) \mathrm{d} s .
\end{aligned}
$$

By Theorem 7.1.1 of Ref. 27 as well as (4.1), (4.3) and (4.6) we conclude that

$$
\|w\| \leq \text { const } t^{-\beta}
$$

for $0<t \leq T$. Finally, taking into account the local existence and (4.13) we finish the proof.

By (4.6), (4.10) and Theorem 3.1 we obtain the main global result

Theorem 4.1. Let initial data (3.6) be such that $\left(w_{0}, v_{0}\right) \in X \times W^{1, \infty}(\Omega)$. If assumptions (2.5), (2.7) are satisfied, then for any $T>0$ problem (3.4)-(3.6) has a unique solution $(w, v)$ in $Y \times Y^{1, \infty}$ and

$$
\begin{aligned}
& w \in C^{1}(] 0, T[; X) \cap C^{0}(] 0, T\left[; W^{2, p}(\Omega)\right), \\
& v \in C^{1}(] 0, T\left[; W^{1, \infty}(\Omega)\right) .
\end{aligned}
$$

Moreover, if $w_{0}, v_{0} \geq 0$, then

$$
w(t) \geq 0, \quad v(t) \geq 0, \quad t \in[0, T] .
$$

Remark 4.1. Theorem 4.1 yields global existence, uniqueness and regularity of solutions to problem (2.8), (2.9), (2.10).

Remark 4.2. By using the Fourier transform techniques one may prove an analogous global existence and uniqueness theorem for Eq. (2.4) with (3.6), $\chi \equiv$ const, in the whole space $\mathbb{R}^{d}$ with the convolution $\star$ instead of $\circledast$, with

$$
u(t) \in H^{l}\left(\mathbb{R}^{d}\right), \quad v(t) \in H^{s}\left(\mathbb{R}^{d}\right), \quad t \in[0, T],
$$

where $l>2+\frac{d}{2}$, and $s>l+1+\frac{d}{2}$ under additional conditions on initial data ensuring $L_{1}$-setting.

Remark 4.3. The regularity of the solutions is strictly related to the regularity of the initial conditions and the regularity of the kernels. In particular, under suitable regularity assumptions on the kernels and on the initial data, we obtain for any $T>0$

$$
\begin{aligned}
& w \in C^{0}([0, T] ; X) \cap C^{1,2}(] 0, T[\times \Omega), \\
& v \in C^{0}\left([0, T] ; C^{1+\alpha}(\bar{\Omega})\right) \cap C^{1}(] 0, T\left[; C^{1+\alpha}(\bar{\Omega})\right),
\end{aligned}
$$

for $0<1+\alpha<2 \beta-d / p$. 


\section{Numerical Analysis and Computational Simulations}

\subsection{Model Nondimensionalisation}

In order to solve the system numerically, we first of all non-dimensionalise the equations. The variables and parameters in the system of equations and their associated boundary conditions are transformed into dimensionless quantities using the following reference variables:

(1) a reference length scale, $L$, (e.g. the maximum invasion distance of the cancer cells at this early stage of invasion $0.1-1 \mathrm{~cm}$ ),

(2) a reference time unit, $\tau=\frac{L^{2}}{D_{c}}$, where $D_{c}$ is a reference chemical diffusion coefficient e.g. $10^{-6} \mathrm{~cm}^{2} \mathrm{~s}^{-1} .{ }^{13}$ Therefore, we deduce that $\tau$ varies between $10^{4}-10^{6}$ sec.

(3) a reference tumour cell density $u_{0}$, extracellular matrix density $v_{0}$ (where $u_{0}$, $v_{0}$ are appropriate reference variables).

We thus define the non-dimensional variables:

$$
\tilde{t}=\frac{t}{\tau}, \tilde{x}=\frac{x}{L}, \tilde{u}=\frac{u}{u_{0}}, \tilde{v}=\frac{v}{v_{0}} .
$$

and new parameters via the following scaling:

$$
\begin{gathered}
\tilde{D}=\frac{D_{u}}{D_{c}}, \tilde{\chi}=\chi_{u} \frac{v_{0}}{D_{c}}, \\
\tilde{\mu}_{1}=\mu_{1} \tau, \tilde{\mu}_{2}=\mu_{2} \tau, \tilde{\gamma}=\gamma u_{0} v_{0} \tau .
\end{gathered}
$$

The haptotactic function is of the form:

$$
\chi(v)=\frac{\tilde{\chi}}{\left(K_{u}+v\right)^{2}}
$$

where $\tilde{\chi} \geq 0$ and $K_{u}>0^{41}$.

Henceforth, we omit the tildes for notational simplicity.

Concerning the numerical analysis and computational simulation of our system, regarding the spatial kernels we proceed as follows (cf. Refs. 14, 15, 26). Let $p \geq 1$. We fix $t \in[0, T]$. Then for each $g(t,.) \in L^{p}(\Omega)$ we consider the linear operator $G: L^{p}(\Omega) \rightarrow W^{2, p}(\Omega)$ where $G(g)$ is the unique solution to the equation

$$
\begin{array}{ll}
-\Delta f+\lambda^{2} f=\lambda^{2} g & \text { in } \Omega, \\
\frac{\partial f}{\partial \nu}=0 & \text { on } \partial \Omega,
\end{array}
$$


for some $\lambda \in \mathbb{R}$. Observe that

$$
f(x)=G(g)(x)=\int_{\Omega} k(x, y) g(y) \mathrm{d} y
$$

satisfies $\nabla f \in W^{1, p}(\Omega)$ and in particular for $d=p=1$ by Sobolev Embedding Theorem (see Ref. 1)

$$
\partial_{x} f \in L^{\infty}(\Omega)
$$

The function $k(x, y)$ is then easily seen to be the Green's Function for the problem

$$
\begin{array}{ll}
-\Delta f+\lambda^{2} f=\delta(x-y) & \text { in } \Omega, \\
\frac{\partial f}{\partial \nu}=0 & \text { on } \partial \Omega,
\end{array}
$$

It is not difficult to see that the assumption guaranteeing the global existence can be be relaxed to cover this case. The general system can be rewritten as

$$
\begin{aligned}
\partial_{t} u(t, x) & =\partial_{x}\left(D \partial_{x} u(t, x)\right)-\partial_{x}\left(\chi(v) u(t, x) \partial_{x} v(t, x)\right) \\
& +\mu_{1} u(t, x)\left(1-f_{1,1}(t, x)-f_{1,2}(t, x)\right) \\
\partial_{t} v(t, x) & =-\gamma v(t, x) f(t, x)+\mu_{2} v(t, x)\left(1-f_{2,1}(t, x)-f_{2,2}(t, x)\right) \\
\lambda^{2} u & =-\partial_{x}^{2} f+\lambda^{2} f \\
\lambda_{i, 1}^{2} u & =-\partial_{x}^{2} f_{i, 1}+\lambda_{i, 1}^{2} f_{i, 1}, \quad i=1,2, \\
\lambda_{j, 2}^{2} v & =-\partial_{x}^{2} f_{j, 2}+\lambda_{j, 2}^{2} f_{j, 2}, \quad j=1,2 .
\end{aligned}
$$

For the computational simulations, we make one final simplification i.e. $k_{1,1}=k_{2,1}$ and $k_{1,2}=k_{2,2}$. This reduces the above system of seven equations to a system of five equations as follows:

$$
\begin{aligned}
\partial_{t} u(t, x) & =\partial_{x}\left(D \partial_{x} u(t, x)\right)-\partial_{x}\left(\chi(v) u(t, x) \partial_{x} v(t, x)\right) \\
& +\mu_{1} u(t, x)\left(1-f_{1,1}(t, x)-f_{2,2}(t, x)\right) \\
\partial_{t} v(t, x) & =-\gamma v(t, x) f(t, x)+\mu_{2} v(t, x)\left(1-f_{1,1}(t, x)-f_{2,2}(t, x)\right) \\
\lambda^{2} u & =-\partial_{x}^{2} f+\lambda^{2} f \\
\lambda_{1,1}^{2} u & =-\partial_{x}^{2} f_{1,1}+\lambda_{1,1}^{2} f_{1,1}, \\
\lambda_{2,2}^{2} v & =-\partial_{x}^{2} f_{2,2}+\lambda_{2,2}^{2} f_{2,2} .
\end{aligned}
$$

Guided by the in vitro experimental protocol in which invasion takes place within an isolated system, we assume no-flux boundary conditions i.e.

$$
\left.u \chi(v) \partial_{x} v-D \partial_{x} u=\partial_{x} f=\partial_{x} f_{1,1}=\partial_{x} f_{2,2}=0, \quad \text { on } \quad\right] 0, T[\times \partial \Omega \text {. }
$$

Finally, the initial distribution of the tumour cells and the extracellular matrix density are prescribed by the system of equations. Initially we assume that there is a cluster of cancer cells already present and that they have penetrated a short 


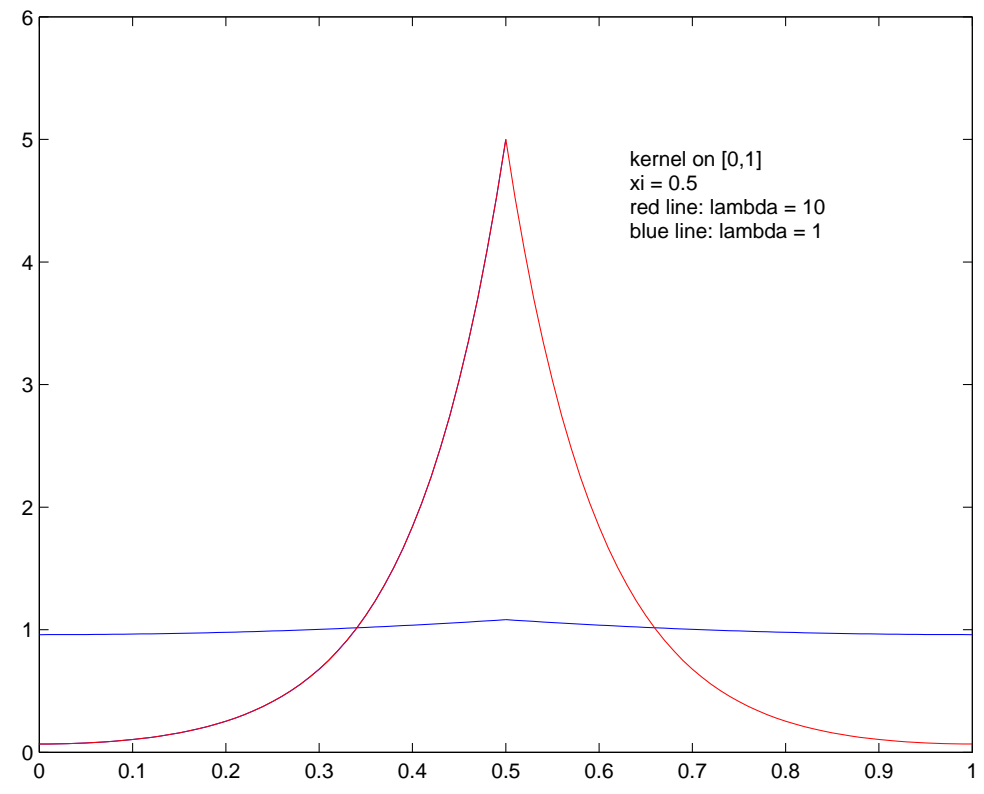

Fig. 1. Plot of the finite kernel (Green's function) on $[0,1], y=0.5$, for the two cases $\lambda=1,10$.

distance into the extracellular matrix while the remaining space is occupied by the matrix alone i.e.

$$
(u(0, x), v(0, x))=\left(u_{0}(x), v_{0}(x)\right)
$$

where

$$
\begin{aligned}
& u_{0}(x)=\exp \left(\frac{-x^{2}}{\epsilon}\right), x \in \Omega \text { and } \epsilon=0.01>0, \\
& v_{0}(x)=1, x \in \Omega
\end{aligned}
$$

For the computational simulation results presented in Section 5.3, we work on a one-dimensional domain $[0, L]$. In this case, it is straightforward to calculate explicitly the Green's Function (and hence the spatial kernel) for our problem (see, for example, Ref. 45). Thus we have

$$
k(x ; y)=\left\{\begin{array}{l}
\frac{\lambda \cosh \lambda x \cosh \lambda(L-y)}{\sinh \lambda L}, 0<x<y \\
\frac{\lambda \cosh \lambda(L-x) \cosh \lambda y}{\sinh \lambda L}, y<x<L
\end{array}\right.
$$


A plot of the above kernel for different values of $\lambda$ is given in figure (5.1). In the above equations we note the role played by the parameters $\lambda$ and $\lambda_{i, j}$ - the smaller $\lambda$ (or $\lambda_{i, j}$ ) is, the greater the nonlocal effect and, conversely, if $\lambda \rightarrow \infty$ the nonlocal term becomes local (see the corresponding equation). The quantity $\frac{1}{\lambda}$ can therefore be considered as a measure of the spatial scale over which the nonlocal term acts. Thus for our system, $\frac{1}{\lambda}$ is a measure of the size of the spatial domain over which degradation acts, $\frac{1}{\lambda_{1,1}}$ is a measure of the size of the spatial domain over which cancer cells compete with each other for space/resources, $\frac{1}{\lambda_{1,2}}$ is a measure of the size of the spatial domain over which cancer cells and components or cells of the ECM compete with each other for space/resources, and so on.

\subsection{Estimation of Parameters}

Whenever possible parameter values are estimated from available experimental data. However, given the large number of parameters in the model to be determined, it is perhaps not surprising that several remain unquantified. In the cases where no experimental data could be found, parameter values were chosen to give the best qualitative numerical simulation results. This is in line with previous papers successfully simulating tumour invasion and angiogenesis. ${ }^{5,16,36}$

Estimation of the Reference Diffusion Coefficients $D_{c}, D_{u}$

We introduce $D_{c}$ a reference chemical diffusion coefficient e.g. $D_{c} \sim 10^{-6} \mathrm{~cm}^{2} \mathrm{~s}^{-1},{ }^{13}$. In their model of epidermal wound healing Sherratt and Murray, used values of $3 \times 10^{-9} \mathrm{~cm}^{2} \mathrm{~s}^{-1}-5.9 \times 10^{-11} \mathrm{~cm}^{2} \mathrm{~s}^{-1}$ for the random motility of epidermal cells. ${ }^{40}$ Furthermore, in their study of individual endothelial cells (ECs), Stokes et al. calculated a random motility coefficient of $(7.1 \pm 2.7) \times 10^{-9} \mathrm{~cm}^{2} \mathrm{~s}^{-1}$ for ECs migrating in a culture containing an angiogenic factor $\alpha \mathrm{FGF}$, heparin and fetal serum as well as a random motility coefficient of migrating endothelial cells with agarose overlays $(2.3 \pm 0.6) \times 10^{-9} \mathrm{~cm}^{2} \mathrm{~s}^{-1}$ and without agarose overlays of $(6.9 \pm 2.6) \times 10^{-9} \mathrm{~cm}^{2} \mathrm{~s}^{-1},{ }^{42}$. In agreement with the aforementioned measurements for cell dispersion Bray estimated the animal cell random motility coefficient to be $\sim 5 \times 10^{-10} \mathrm{~cm}^{2} \mathrm{~s}^{-1},{ }^{13}$. In this regard, our choice for the cell random motility coefficient $D_{u}$ will vary betweeen $10^{-9} \mathrm{~cm}^{2} \mathrm{~s}^{-1}$ and $10^{-11} \mathrm{~cm}^{2} \mathrm{~s}^{-1}$, so our nondimensional value will be between: $D=10^{-3}-10^{-5}$.

\section{The haptotactic coefficient $\left(\chi_{u}\right)$}

Stokes et al. estimated the chemotaxis coefficient of ECs migrating in a culture containing $\alpha \mathrm{FGF}$, to be $2600 \mathrm{~cm}^{2} \mathrm{~s}^{-1} M^{-1},{ }^{42}$. A value of $v_{0}$ in the range $0.38 \times$ 
$10^{-9} M-0.38 \times 10^{-12} M$ is consistent with experimental measurements. In the absence of reliable empirical data, we chose the haptotaxis coefficient $\chi_{u}$ to be in the range of $2.5 \times 10^{-3}-2.5 \times 10^{-1} \mathrm{~cm}^{2} \mathrm{~s}^{-1} \mathrm{M}^{-1}$. Therefore, considering the fact that the vitronectin blood plasma concentration is around $4 \mu M,{ }^{20}$ leads to a dimensionless estimate of the haptotaxis coefficient $\chi$ in the range between 0.001 1.

Proliferation rate constant, $\mu_{\mathbf{1}}$

$\mathrm{Yu}$ et al. estimated the doubling time of human epidermoid carminoma cells (HEp3) from in vitro proliferation experiments time to be $24 h .^{44}$ By taking the proliferation rate as the reciprocal of the cell-cycle time we get $\tilde{\mu}_{1} \sim 0.042 h^{-1}$.

In this regard, in our numerical simulations we will choose the proliferation rate to be between $0.02 h^{-1}-0.72 h^{-1}$, and thus obtain the dimensionless parameter of $\mu_{1}$ in the range $0.05-2$.

Parameters $\lambda_{i}, \quad i=1,2,3$

As noted previously, the quantities $1 / \lambda_{i}$ can be considered as a measure of the spatial scale over which the nonlocal term acts. In our simulations, we used values of $\lambda_{1}=1-10$ in dimensionless units. Although the cancer cells have rather nonregular shape we estimate that its average diameter is equal to 10 microns. The above range for $\lambda_{1}$ is therefore equivalent to the assumption that the non-local effect varies from 1 cell diameter up to 10 cell diameters. Similarly we used values of $\lambda_{2}=10-50$ and $\lambda_{3}=1-10000$.

\section{Remaining Parameters}

Not all parameters in the model were able to be estimated. Therefore, we chose these values in order to give the best qualitative results in the simulations. For the extracellular matrix degradation rate we consider $\gamma$ to vary between $1-20$. We took $\mu_{2}$ in the range of $0.15-2.5$. 


\begin{tabular}{lll}
\hline Parameter & Description & Value \\
\hline$D$ & cell diffusion coefficient & $10^{-5}-10^{-3}$ \\
$\chi$ & haptotactic coefficient & $10^{-3}-1$ \\
$\mu_{1}$ & proliferation rate of cancer cells & $5 \cdot 10^{-2}-2$ \\
$\mu_{2}$ & matrix re-modelling rate & $1.5 \cdot 10^{-1}-2.5$ \\
$\gamma$ & matrix degrading rate & $1-2 \cdot 10$ \\
$\lambda_{1}$ & cancer cell non-local effect & $1-10$ \\
$\lambda_{2}$ & ECM non-local effect & $1-50$ \\
$\lambda_{3}$ & degradation non-local effect & $1-10000$ \\
\hline
\end{tabular}

\subsection{Computational Simulations}

We now present some computational results from numerical simulations of our system of equations 5.7. Figures 2 and 3 show the results from a computational simulation of equations (5.6). In this case the parameters $\lambda_{1}=10, \lambda_{2}=50$ and $\lambda_{3}=10000$. The choice of parameter $\lambda_{3}=10000$ means in effect that the degradation term of the second equation of (5.6) is almost local i.e. $\approx-\gamma u v$. We chose this value for $\lambda_{3}$ in order to focus on the effect of nonlocal proliferation. As can be seen from the plots in figures 2 and 3, the initial profile of cancer cells develops into a travelling wave which invades the ECM, degrading the ECM as it invades. Eventually all the ECM is degraded and we are left with the cancer-only steady state of $(1,0)$.

Figures 4 and 5 show the results from a computational simulation of equations (5.6) where the parameters $\lambda_{1}=1, \lambda_{2}=5$ and $\lambda_{3}=10000$. As can be seen from both sets of plots, once again the initial profile of cancer cells develops into a travelling wave which invades the ECM, degrading the ECM as it invades. However, in this case we note that there is a "bump" in the cancer cell density at the front of the travelling wave, where the maximum cancer cell density reaches a value of approximately 1.5. This is due to the influence of the nonlocal proliferation terms whose effects have been enhanced due to the choice of parameters $\lambda_{1}=1, \lambda_{2}=5$. Once again, the wave of cancer cells invades the ECM, degrading the ECM as it invades. Eventually all the ECM is degraded and we are left with the cancer-only steady state of $(1,0)$. We note that in this case, the cancer cells penetrate less deeply into the ECM - at $t=60$ the leading edge of the cancer cells has reached the point just beyond $x=0.7$, while in figure 2 at $t=60$ the leading edge of the cancer cells has reached approximately $x=0.625$.

The results presented in figures 2,3 and 4,5 illustrate the effect of the nonlocal proliferation terms. The cancer cells degrade the surrounding tissue and then invade this degraded region of tissue by a combination of diffusion (random motility) and haptotaxis. Mathematically this is seen as the travelling wave solution connecting the cancer-free state with the cancer-only state. In the model we assume that cancer cells are competing for nutrient (e.g. oxygen) with other cells at different spatial locations. In the one-dimensional domain considered here this means that the cancer 


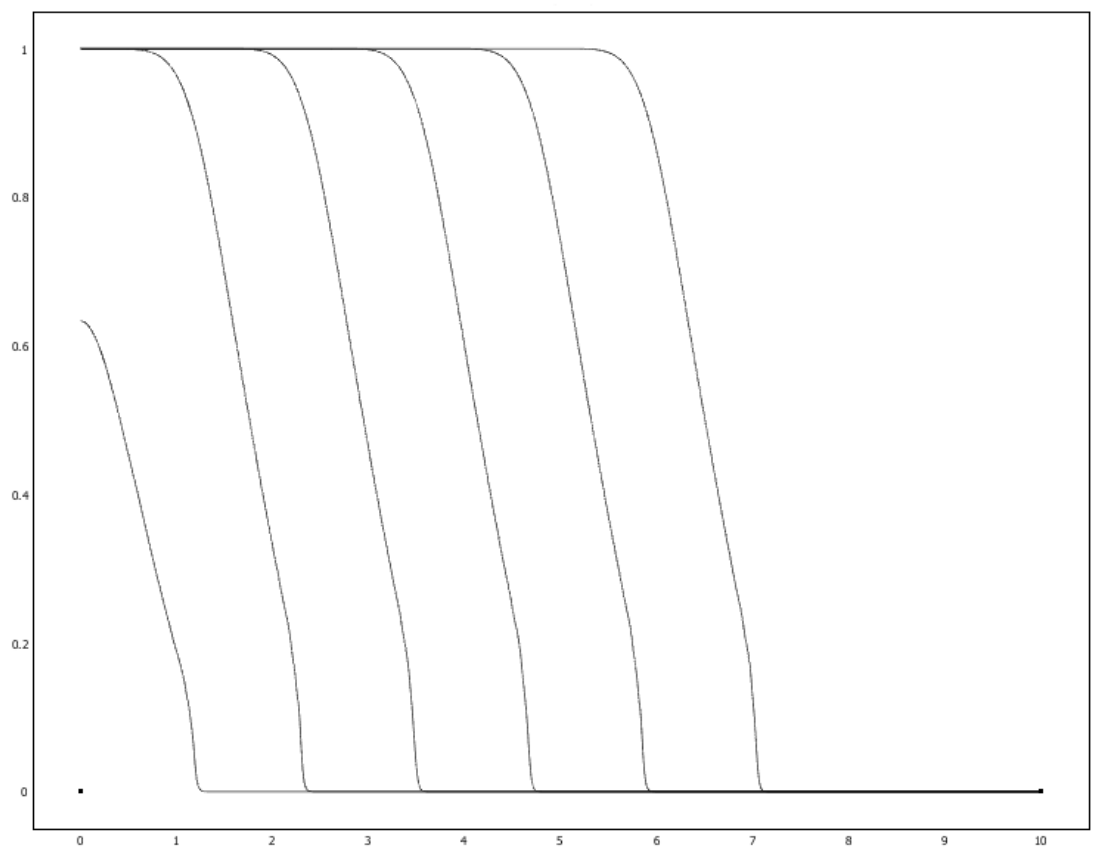

Fig. 2. Plot showing profile of the density of cancer cells at times $t=10,20,30,40,50,60$. Figure shows the travelling wave of invasion of cancer cells invading the ECM. Parameters $\lambda_{1}=10, \lambda_{2}=$ $50, \lambda_{3}=10000, D=0.001, \chi=0.075, \mu_{1}=1, \mu_{2}=0.15$ and $\gamma=1$.

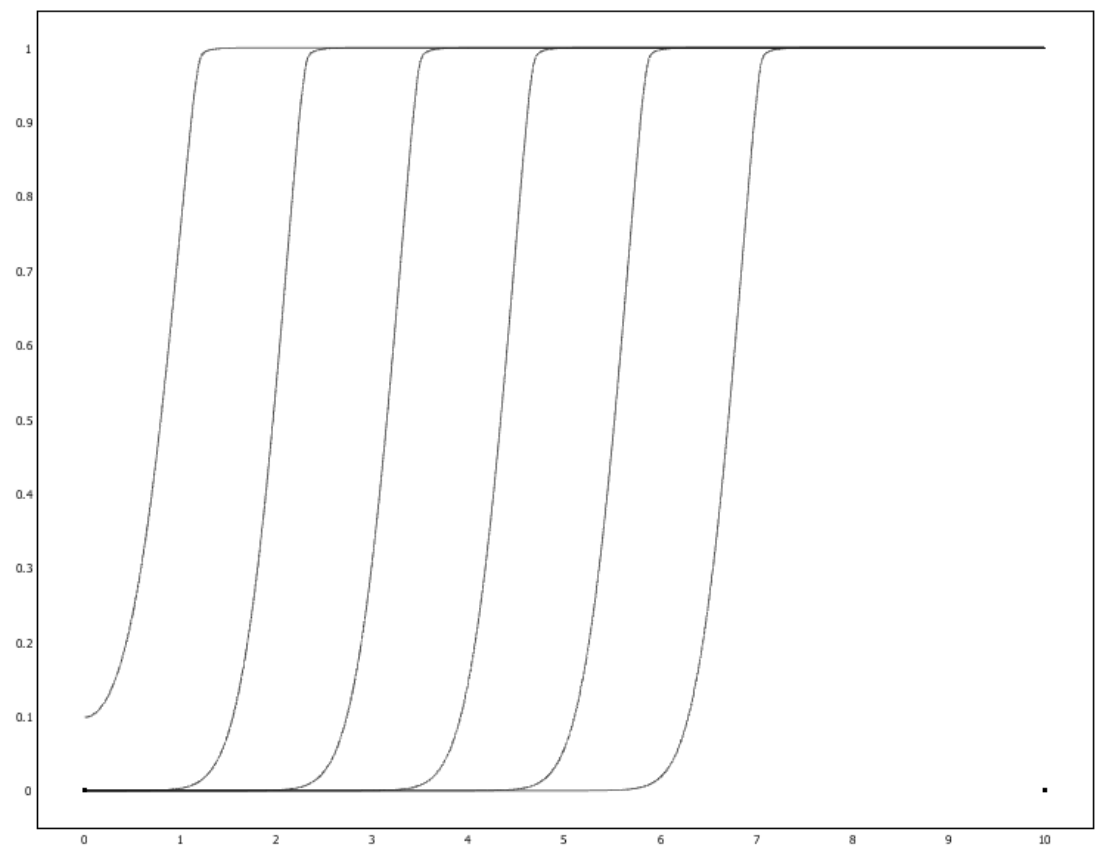

Fig. 3. Plot showing the corresponding profile of the density of ECM at times $t=$ 10, 20, 30, 40, 50,60. Figure shows ECM being degraded by the cancer cells as they invade. 


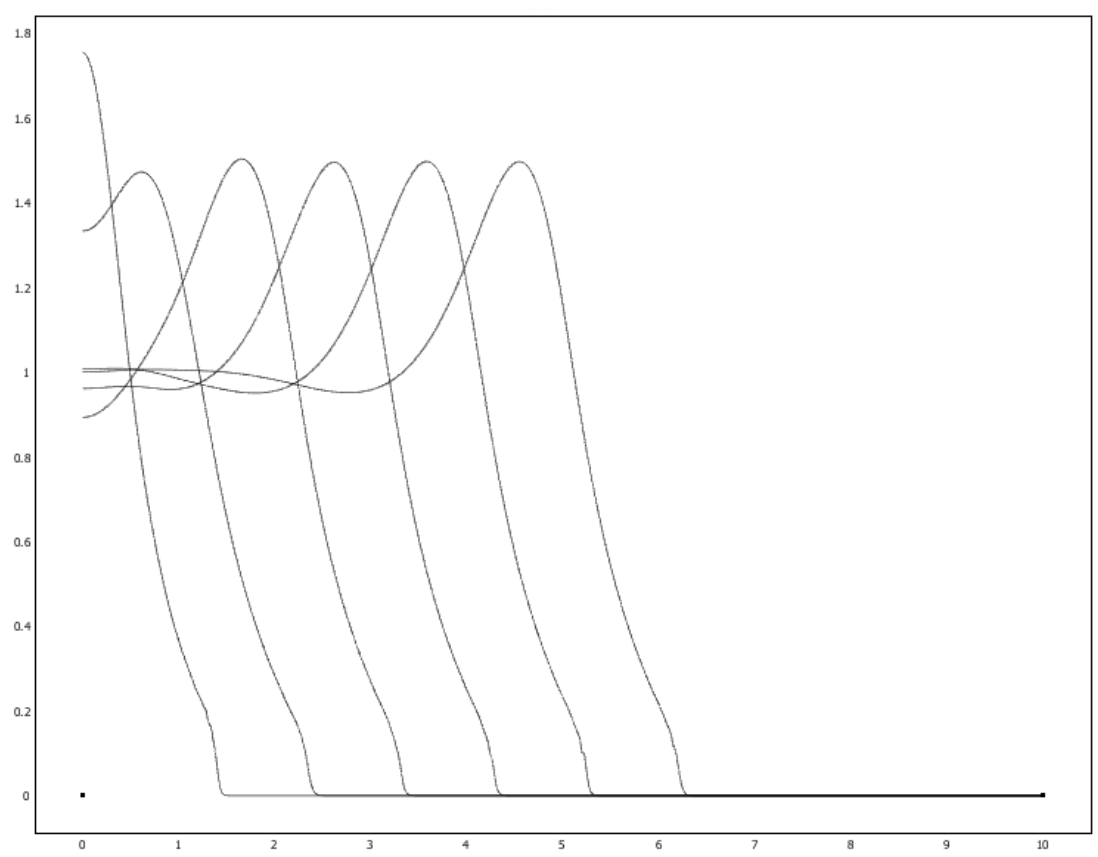

Fig. 4. Plot showing profile of the density of cancer cells at times $t=10,20,30,40,50,60$. Figure shows the travelling wave of invasion of cancer cells invading the ECM. Parameters $\lambda_{1}=1, \lambda_{2}=$ $5, \lambda_{3}=10000, D=0.001, \chi=0.075, \mu_{1}=1, \mu_{2}=0.15$ and $\gamma=1$.

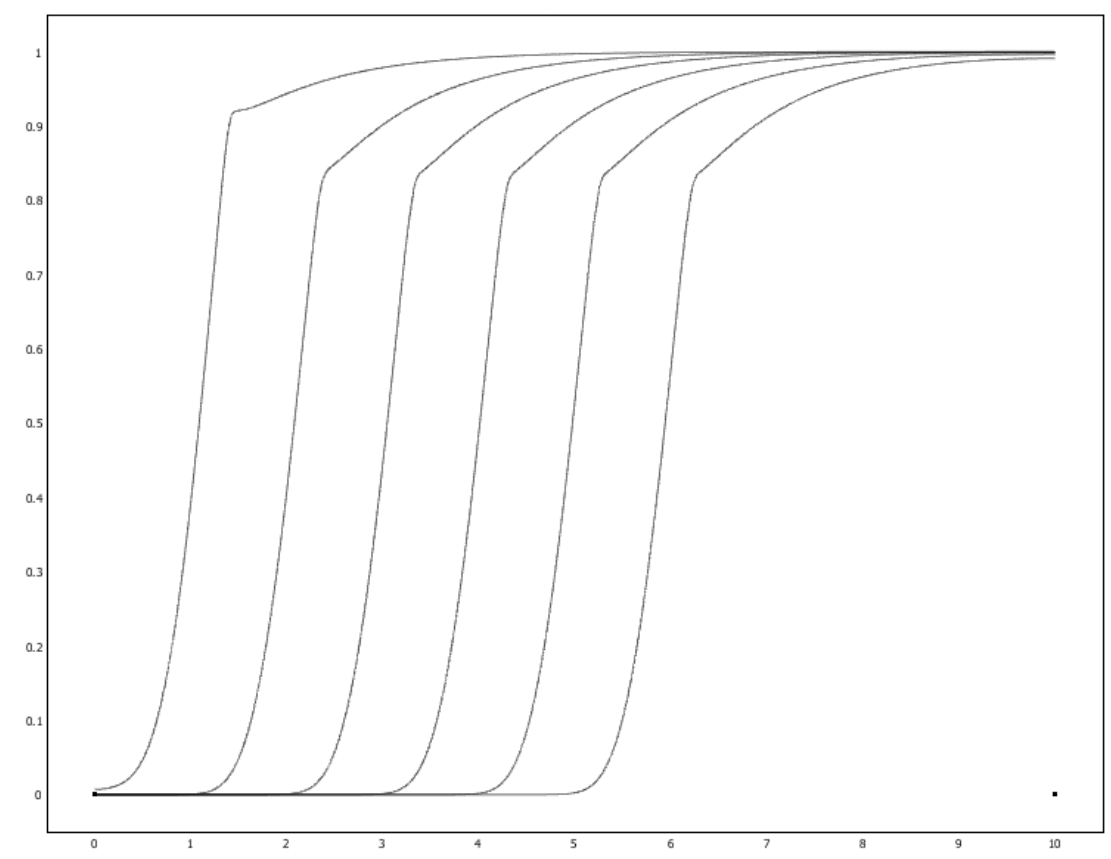

Fig. 5. Plot showing the corresponding profile of the density of ECM at times $t=$ 10, 20, 30, 40, 50, 60. Figure shows ECM being degraded by the cancer cells as they invade. 
cells competing both with those cells in front and with those cells behind. In an invasion of a region of tissue where there were no cancer cells, those cells at the front of the invasion wave (mathematically, at the point where the travelling front is steepest) will find there are essentially no cancer cells ahead of them. This means that essentially these cells are only in competition with the cells behind. This gives them an "invasive advantage" and allows the cell numbers there to get above the carrying capacity level (the maximum level that can be sustained in the long term), but only in the neighbourhood of the front of the invading cells. ${ }^{26}$ As a result we see a hump in the front profile.

\section{Discussion}

In this paper we have presented a mathematical model of cancer cell invasion of tissue and investigated the effect of nonlocal reaction kinetics. The model was formulated as a system of partial differential equations (integro-differential equations) with the nonlocal terms modelling competition for nutrient between the cancer cells and tissue re-modelling. Additionally we incorporated a nonlocal degradation term. Certain important analytical results were proved and computational results of numerical simulations of our model were given.

In Section 3 we presented a mathematical analysis of the model and proved some existence results for the solution of our equations using the theory of linear semigroups. In Section 4 we proved that the solutions to the nonlocal equations exist globally in any space dimension $d$ without imposing any kind of smallness conditions in the initial conditions. Specifically by using Fourier transform techniques we proved a global existence and uniqueness theorem. Finally regarding a formal analysis of our system, we showed that the regularity of the solutions is strictly related to the regularity of the initial conditions and the regularity of the kernels and proved some results related to this.

In Section 5 we presented the computational results of numerical simulations of our basic model. The results of these simulations showed the effect of the nonlocal terms. ${ }^{26}$ Travelling waves of invading cancer cells were observed, and the shape of the travelling wave was closely linked to the nonlocal terms and the size of the parameters $\lambda_{i}$. The invasive waves were either "regular" or had a "hump" at the front, indicating a region of high cancer cell density. From a biological perspective these results indicate the important role that competition for nutrient (e.g. oxygen) and space may play during cancer cell invasion. The results of the numerical simulations indicate that cancer cell at the leading edge of an invasive front are only in competition with the cells behind, giving them an "invasive advantage" over cells futher behind. This may have implications for the depth of penetration into the ECM.

In addition to proliferation, migration and competition for space, cell-cell and cell-matrix adhesion are other vitally important features of cancer invasion. Until 
now, this effect has only really been investigated using discrete models,${ }^{23,24,6}$ although recent work on cell-cell and cell-matrix adhesion using a continuum PDE model has been developed by Armstrong et al. (see Ref. 9). Future work will consider using nonlocal terms to more accurately model cell-cell and cell-matrix adhesion.

\section{Acknowledgment:}

The authors gratefully acknowledge support from the EU Marie Curie Research Training Network Grant "Modelling, Mathematical Methods and Computer Simulations of Tumour Growth and Therapy", contract number MRTN-CT-2004-503661 and Polish SPUB-M. The work of ZS was partially supported by the Polish-German PhD studies Graduate College "Complex Processes: Modelling, Simulation and Optimization". The authors thank Professor Maciej Żylicz and Jakub Urbański from Molecular Biology Department at IIMCB for helpful advice and comments.

\section{References}

1. R. A. Adams, Sobolev Spaces, Academic Press, New York (1975)

2. D. Albo, D. H. Berger, V. L. Rothman and G. P. Tuszynski, Role of urokinase plasminogen activator receptor in thrombospondin 1-mediated tumor cell invasion, J. Surg. Res. 82 (1999) $331-338$.

3. H. Amann, Dynamic theory of quasilinear parabolic equations II. Reaction-diffusion systems, Differ. Integral Eq. 3 (1990) 13-75.

4. H. Amann, Nonhomogeneous Linear and Quasilinear Elliptic and Parabolic Boundary Value Problems, Eds. Schmeisser and Triebel, Function Spaces, Differential Operators and Nonlinear Analysis, Teubner Texte zur Mathematik, 133 (1993) 9-126.

5. A. R. A. Anderson, M. A. J. Chaplain, E. L. Newman, R. J. C. Steele and A. M. Thompson, Mathematical modelling of tumour invasion and metastasis, J. Theor. Med. 2 (2000) $129-154$.

6. A. R. A. Anderson, A hybrid mathematical model of solid tumour invasion: The importance of cell adhesion. Math. Med. Biol. 22 (2005) $163-186$.

7. P. A. Andreasen, L. Kjøller, L. Christensen and M. J. Duffy, The urokinase-type plasminogen activator system in cancer metastasis: A review, Int. J. Cancer $\mathbf{7 2}$ (1997) $1-22$.

8. P. A. Andreasen, R. Egelund and H. H. Petersen, The plasminogen activation system in tumor growth, invasion, and metastasis, Cell. Mol. Life Sci. 57 (2000) $25-40$.

9. N.J. Armstrong, K. Painter and J.A. Sherratt, A continuum approach to modelling cell-cell adhesion, J. Theor. Biol. 243 (2006) $98-113$.

10. S. Aznavoorian, M. L. Stracke, H. Krutzsch, E. Schiffmann and L. A. Liotta, Signal transduction for chemotaxis and haptotaxis by matrix molecules in tumor cells, $J$. Cell Biol. 110 (1990) 1427 - 1438.

11. S. Aznavoorian, A. N. Murphy, W. G. Stetler-Stevenson and L. A. Liotta, Molecular aspects of tumor cell invasion and metastasis, Cancer 71 (1993) 1368 - 1383.

12. S. Aznavoorian, M. L. Stracke, J. Persons, J. McClanahan and L. A. Liotta, Integrin $\alpha_{V} \beta_{3}$ mediates chemotactic and haptotactic motility in human melanoma cells through different signaling pathways, J. Biol. Chem. 271 (1996) 3247 - 3254.

13. D. Bray, Cell Movements: From Molecules to Motility, Garland Publishing (2000).

14. N.F. Britton, Aggregation and the competitive exclusion principle, J. Theor. Biol. 136 (1989) $57-66$. 
15. N.F. Britton, Spatial structures and periodic travelling waves in an integrodifferential reaction-diffusion population model, SIAM J. Appl. Math., 50 (1990) 1663 - 1688.

16. H. M. Byrne, M. A. J. Chaplain, G. J. Pettet and D. L. S. McElwain, A mathematical model of trophoblast invasion, J. Theor. Med. 1 (1998) $275-286$.

17. S. B. Carter, Haptotaxis and the mechanism of cell motility, Nature 213 (1967) 256 -260 .

18. M. A. J. Chaplain and G. Lolas, Mathematical modelling of cancer cell invasion of tissue: The role of the urokinase plasminogen activation system, Math. Mod. Meth. Appl. Sci. 15 (2005) 1685 - 1734.

19. M. A. J. Chaplain and G. Lolas, Mathematical modelling of cancer invasion of tissue: Dynamic heterogeneity, Net. Hetero. Med. 1 (2006) $399-439$.

20. W. D. Comper, Extracellular Matrix Volume 2 Molecular Components and Interactions, Hartwood Academic (1996).

21. L. Corrias, B. Perthame and H. Zaag, Global solutions of some chemotaxis and angiogenesis systems in high space dimensions, Milan J. Math. 72 (2004) 1-28.

22. A. S. G. Curtis, The measurement of cell adhesiveness by an absolute method, J. Embryol. Exp. Morphol. 22 (1969) $305-325$.

23. D. Drasdo and S. Höhme, A single-cell-based model of tumor growth in vitro: monolayers and spheroids, Phys. Biol., 2 (2005) 133 - 147.

24. J. Galle, G. Aust, G. Schaller, T. Beyer and D. Drasdo, Individual cell-based models of the spatio-temporal organisation of multicellular systems - achievements and limitations, Cytometry.

25. R. A. Gatenby and E. T. Gawlinski, A reaction-diffusion model of cancer invasion, Cancer Res. 56 (1996) 5745 - 5753.

26. S.A. Gourley, M.A.J. Chaplain and F.A. Davidson, Spatio-temporal pattern formation in a nonlocal reaction-diffusion equation, Dyn. Sys. 16 (2001) 173 - 192.

27. D. Henry, Geometric theory of semilinear parabolic equations, Lecure Notes Math. 840, Springer 1981.

28. T. Hillen, K. Painter, Global existence for a parabolic chemotaxis model with prevention of overcrowding, Adv. Appl. Math. 26 (2001) 280-301.

29. D. Horstmann, From 1970 until present: the Keller-Segel model in chemotaxis and its consequences. I. Jahresber. Deutsch Math.-Verein. 105 (2003) 103-165.

30. D. Horstmann, From 1970 until present: the Keller-Segel model in chemotaxis and its consequences. II. Jahresber. Deutsch Math.-Verein. 106 (2004) 51-69.

31. M. Lachowicz, Micro and meso scales of description corresponding to a model of tissue invasion by solid tumours, Math. Mod. Meth. Appl. Sci. 15 (2005) $1667-$ 1683

32. D. A. Lauffenburger and A. F. Horwitz, Cell migration: A physically integrated molecular process, Cell 84 (1996) 359 - 369.

33. J. B. McCarthy, S. L. Palm and L. T. Furcht, Migration by haptotaxis of a Schwann cell tumor line to the basement membrane glycoprotein laminin, J. Cell Biol. 97 (1983) $772-777$.

34. J. B. McCarthy, S. T. Hagen and L. T. Furcht, Human fibronectin contains distinct adhesion- and motility-promoting domains for metastatic melanoma cells, J. Cell Biol. 102 (1986) $179-188$.

35. M. E. Orme and M. A. J. Chaplain, A mathematical model of vascular tumour growth and invasion, Math. Comput. Model. 23 (1996) $43-60$.

36. M. E. Orme and M. A. J. Chaplain, Two-dimensional models of tumour angiogenesis and anti-angiogenesis strategies, IMA J. Math. Appl. Med. Biol. 14 (1997) 189 - 205. 
37. S. L. Parsons, S. A. Watson, P. D. Brown, H. M. Collins and R. J. C. Steele, Matrix metalloproteinases, Brit. J. Surg. 84 (1997) $160-166$.

38. A. J. Perumpanani, J. A. Sherratt, J. Norbury, H. M. Byrne, Biological inferences from a mathematical model for malignant invasion, Invasion $\&$ Metastasis 16 (1996) $209-221$.

39. A. J. Perumpanani, D. L. Simmons, A. J. H. Gearing, K. M. Miller, G. Ward, J. Norbury, M. Schneemann and J. A. Sherratt, Extracellular matrix-mediated chemotaxis can impede cell migration, Proc. Roy. Soc. London, Ser. B 265 (1998) 2347 2352 .

40. J. A. Sherratt and J. D. Murray, Models of epidermal wound healing, Proc. Roy. Soc. London, Ser. B 241 (1990) 29 - 36.

41. J. A. Sherratt, Chemotaxis and chemokinesis in eukaryotic cells: the Keller-Segel equations as an approximation to a detailed model, Bull. Math. Biol. 56 (1994) 129-146.

42. C. L. Stokes and D. Lauffenburger, Analysis of the roles of microvessel endothelial cell random motility and chemotaxis in angiogenesis, J. Theor. Biol. 152 (1991) 377 - 403.

43. G. Taraboletti, D. D. Roberts and L. A. Liotta, Thrombospondin-induced Tumor Cell Migration: Haptotaxis and Chemotaxis are Mediated by Different Molecular Domains, J. Cell Biol. 105 (1987) 2409 - 2415.

44. W. Yu, J. Kim and L. Ossowski, Reduction in surface urokinase receptor forces malignant cells into a protracted state of dormancy, J. Cell Biol. 137 (1997) 767 777.

45. E. Zauderer, Partial Differential Equations of Applied Mathematics, Second Edition, (John Wiley \& Sons, 1989). 